\title{
Comparação do Pico de Fluxo Expiratório e a Cirtometria Torácica entre Idosas, Antes e Após a Realização de Fisioterapia Respiratória
}

\section{Comparison of Expiratory Flow Peak and Thoracic Cirtometry Between Elderly People, Before and After Respiratory Physiotherapy}

\section{RESUMO}

Objetivo: Este estudo visou comparar o Pico de fluxo expiratório (PFE) e a Cirtometria torácica de idosas não institucionalizadas e institucionalizadas, antes e após a realização de exercícios respiratórios. Metodologia: Após o cálculo amostral, 21 mulheres com idade compreendida entre 65 e 85 anos $(74,71 \pm 6,47$ anos) foram submetidas à realização da espirometria e da cirtometria torácica, antes e após a realização dos exercícios respiratórios. Foram realizadas oito visitas, com as voluntárias divididas em dois grupos. As médias e os desvios padrões foram utilizados para a análise descritiva e para normalidade dos dados, por meio do teste de Shapiro-Wilk. Comparou-se as médias pré vs. pós a realização das oito sessões com o teste ANOVA de dois caminhos e medidas repetidas, para identificar a interação no fator tempo. O nível de significância adotado foi de $\alpha=0,05$ e análises foram realizadas no software SPSS 17.0 for Windows ${ }^{\circledR}$. Resultados: O PFE pré vs. pós realização dos exercícios respiratórios, apresentou diferenças significativas $(p<0,05)$ e a cirtometria, ainda que apontando diferenças entre os grupos, não sofreu alterações após os exercícios. Conclusão: Portanto, o PFE dos grupos foi aumentado durante o programa de exercícios respiratórios em oito sessões e para os valores da cirtometria torácica não ocorreram alterações. Contudo, as idosas institucionalizadas apresentaram médias de expansão e retração torácica menor do que as não institucionalizadas.

\section{DESCRITORES}

Idoso. Espirometria. Mecânica Respiratória. Músculos Respiratórios.

\begin{abstract}
Objective: This study aimed to compare the Expiratory Flow Peak (PEF) and Thoracic Cirtometry of non-institutionalized and institutionalized elderly women before and after respiratory exercises. Methodology: After the sample calculation, 21 women aged $65-85$ years $(74,71 \pm 6,47$ years) were submitted to Spirometry and Thoracic Cirtometry, before and after the performance of respiratory exercises. Eight visits were conducted, with volunteers divided into two groups. The means and standard deviations were used for descriptive analysis and the Shapiro-Wilk test for the normality of the data. We compared the pre vs. post performing the eight sessions with two-way ANOVA and repeated measures, to identify the interaction in the time factor. The significance level adopted was $\alpha=0,05$ and analyses were performed in SPSS 17.0 for Windows $₫$ software. Results: The PEF pre vs. post the completion of respiratory exercises, presented significant differences $(p<0,05)$ and Cirtometry, although showing differences between the groups, did not change after exercise. Conclusion: Therefore, the PEF of the groups was increased during the respiratory exercise program in eight sessions and there were no changes in the values of Thoracic Cirtometry. However, institutionalized elderly women presented a mean of expansion and lower thoracic retraction than those not institutionalized.
\end{abstract}

\section{DESCRIPTORS}

Elderly. Spirometry. Respiratory Mechanics. Respiratory Muscles.

\footnotetext{
${ }_{1}^{1}$ Fisioterapeuta, graduado pela Universidade Presidente Antônio Carlos/UNIPAC. Barbacena, Minas Gerais, Brasil.

${ }^{2}$ Fisioterapeuta, Professora e Coordenadora do Curso de Fisioterapia da Universidade Presidente Antônio Carlos/UNIPAC. Barbacena, Minas Gerais, Brasil.
} 
envelhecimento é um evento fisiológico ${ }^{1}$ que pode causar em idosos inúmeras incapacidades, onde ações preventivas, curativas, de promoção e de reabilitação, podem ser capazes de promover o aumento da capacidade funcional, da autonomia, da independência e, consequentemente, da melhora da qualidade de vida ${ }^{2}$. Atualmente, ele vem sendo descrito como um fator crescente no Brasil, que ocupa a sétima posição no ranking de países com maior número de idosos do mundo e espera-se que em 2025 atinja a sexta posição, devido ao declínio das taxas de fecundidade e do aumento da longevidade ${ }^{3-5}$.

Alterações e morbidades que acompanham o envelhecimento, como na função respiratória, podem estar conjugadas com o aparecimento de maior resistência das vias aéreas e da diminuição da complacência da caixa torácica e pulmonar ${ }^{6}$. Estas alterações podem resultar na limitação da função pulmonar, levando ao declínio do volume expiratório máximo (VEM), da capacidade vital (CV) em aproximadamente $25 \%$ a $40 \%$, no aumento da capacidade residual funcional (CRF) e do volume de reserva expiratório (VRE) ${ }^{7}$. Consequentemente, provocam à redução do pico de fluxo expiratório (PFE), que é apontado como um fator de desequilíbrio para a homeostase respiratória ${ }^{8}$.

Este desequilíbrio respiratório pode ser maior em idosas institucionalizadas, devido à acentuada redução da massa muscular, em especial, do músculo diafragma e dos músculos acessórios da respiração ${ }^{9}$, que ocasionam para esta população, menores valores de PFE, de medidas de pressão inspiratória máxima (PImax) e de pressão expiratória má- xima $(\text { PEmax })^{10}$, podendo levar a movimentos anormais durante a respiração e a diminuição da troca de ar na superfície alveolar ${ }^{11}$. Sendo assim, o método da cirtometria pode ser uma técnica útil para mensurar a mobilidade torácica ${ }^{12,13}$ e a espirometria, uma técnica simples de medidas respiratórias, para se mensurar a entrada e saída de ar dos pulmões ${ }^{14,15}$, com objetivo de avaliar a capacidade pulmonar e o efeito da conduta respiratória, auxiliando fisioterapeutas, tanto na prescrição de um programa de tratamento como para um programa de prevenção ${ }^{16}$.

Uma vez que a espirometria e a cirtometria são descritas como instrumentos de medidas de boa confiabilidade e que alterações do PFE, da expansibilidade e da retração torácica, podem estar presentes nas idosas, este estudo visou a comparação destes valores em idosas institucionalizadas e idosas não institucionalizadas, antes e após a realização de exercícios respiratórios, uma vez que a institucionalização pode ser um fator que leva os indivíduos a terem um baixo condicionamento físico, fazendo com que os músculos respiratórios fiquem enfraquecidos ${ }^{6}$.

\section{METODOLOGIA}

O projeto foi submetido ao Comitê de Ética em Pesquisa (CEP) da Universidade Presidente Antônio Carlos (UNIPAC) e foi aprovado por meio do Parecer $n^{\circ}$. 2.053.430, com data de relatoria em 09 de maio de 2017. O estudo encontra-se registrado no Ministério da Saúde, por meio do Registro Brasileiro de Ensaios Clínicos, sob o identificador primário RBR-3xfyhh. As voluntárias foram convidadas a participar da pesquisa, através de convites 
orais, realizados pelos pesquisadores responsáveis nas dependências da Clínica Escola Vera Tamm de Andrada e do Instituto Mauro Alcides Ferreira, no Município de Barbacena/ MG.

Após o convite, as idosas que compareceram, receberam informações detalhadas sobre os procedimentos do estudo e esclarecimentos sobre as visitas a que seriam submetidas, estando cientes desde o princípio, que a qualquer momento poderiam retirar-se da pesquisa, sem nenhum ônus ou prejuízo em função de suas decisões. Em seguida, elas receberam o Termo de consentimento livre e esclarecido e o Questionário estratificado de risco cardiovascular, que descreviam sobre todos os procedimentos do estudo e classificavam o risco para a realização do protocolo de exercícios.

O cálculo amostral foi realizado com base em um estudo-piloto, que envolveu a participação de 14 voluntários e utilizou-se a equação proposta por Hopkins ${ }^{17}$ para desenhos experimentais de pesquisa clínica, de segmento transversal, observacional, prospectivo, comparativo, analítico, a fim de alcançar um poder estatístico $(1-\beta)$ de 0,80 .

Após a análise estatística sobre o estudo-piloto, foi observado que a amostra seria por conveniência, devido a acessibilidade e população específica disponível, portanto, foi possível obter um número total de amostragem de 21 indivíduos do sexo feminino, com idade entre 65 e 85 anos (74,71 26,47 anos).

As idosas foram distribuídas em dois grupos, sendo 14 não institucionalizadas (GINI) e 07 institucionalizadas (GII). Optou-se por desenvolver a pesquisa com mulheres, buscando a homogeneização do estudo e por este gênero ter demonstrado, segundo o Instituto Brasileiro de Geografia e Estatística (IBGE), no Município de Barbacena/MG, maiores índices de mortalidade -43 óbitos (55\%), comparados aos homens - 35 óbitos (45\%), por doenças respiratórias ${ }^{18}$. A amostra foi composta pelas voluntárias que se propuseram a participar da pesquisa, sendo desconsiderados apenas aquelas que não se adequaram aos critérios de inclusão e exclusão.

Foram empregados como critérios de inclusão, idosas cooperativas ${ }^{16,19}$, com boa cognição ${ }^{20}$, conscientes ${ }^{19}$, portadoras de doenças cardíacas, metabólicas, renais e hepáticas, obesas, sedentárias ${ }^{19}$, que responderam ao Questionário estratificado de risco cardiovascular (QERC) e que concordaram em assinar o Termo de consentimento livre e esclarecido (TCLE). Como critérios de exclusão utilizou-se a idade inferior a 65 e maior que 85 anos, com déficit cognitivo elevado ${ }^{7,10,16,20-22,23-25}$, traqueostomizadas $^{19}$, fumantes ${ }^{7,10,19,24,26,27-29}$, portadoras de doenças respiratórias ${ }^{7,8,16,21,22,28-30} \mathrm{e}$ neurológicas ${ }^{10,23,25,30,31}$, cadeirantes ${ }^{16,21,25,28,29}$, acamadas ${ }^{16}$, com demência e que expuseram quaisquer condições que as impossibilitaram de realizar os exercícios propostos.

Logo, duas idosas foram excluídas do estudo, porém, além dos critérios definidos acima, quatro das participantes optaram por não continuar como voluntárias da pesquisa e, portanto, foram contabilizadas como exclusão.

Para coleta dos dados, foram realizadas oito visitas, duas vezes por semana, na Clínica Escola Vera Tamm de Andrada, da Universidade Presidente Antônio Carlos - UNIPAC/Campus Barbacena, situada à Rodovia MG, 338, km 12, s/nº - Colônia Rodrigo Silva, Barbacena/MG e no Instituto Mauro 
Alcides Ferreira (IMAF), localizado na Rua Santa Catarina, $n^{\circ} 300$, Bairro Funcionários, em Barbacena/MG.

Na primeira visita, com duração de 60 minutos, as participantes receberam orientações sobre os procedimentos, riscos, benefícios e tiraram todas as dúvidas em relação aos exercícios respiratórios, à espirometria e à cirtometria. Após, foi realizada a leitura do TCLE por um dos pesquisadores e, as que concordaram em participar, responderam ao QERC e assinaram de forma física ou com impressão digital o TCLE. Logo depois, realizaram a primeira coleta de sinais vitais, medidas da cirtometria e espirometria.

Da segunda à sétima visita, com duração de 60 minutos, as idosas deram início ao programa de exercícios propostos que foi dividido em duas fases: inicial e final. A fase inicial foi composta por exercícios diafragmáticos ${ }^{31}$, inspiração fracionada em tempos, com expiração freno-labial ${ }^{32}$ e ventilação padrão, com expiração curta ${ }^{31}$, onde foram orientadas a ficarem na posição sentada, em uma cadeira, com roupas e sapatos confortáveis. Posteriormente, na fase final, as idosas realizaram os exercícios respiratórios lúdicos ${ }^{33}$, com duração de 10 minutos, na posição ortostática.

Inicialmente, as participantes foram orientadas a permanecerem sentadas, para coleta da espirometria e dos sinais vitais, sendo: frequência cardíaca (FC); saturação de oxigênio $\left(\mathrm{SaO}_{2}\right)$; frequência respiratória (FR); pressão arterial (PA). Em seguida, as variáveis foram verificadas com o intuito de minimizar os riscos de intercorrências hemodinâmicas durante os exercícios, sendo permitido que as idosas realizassem as atividades, desde que fossem observadas as condições estabe- lecidas na I Diretriz Brasileira de Prevenção Cardiovascular ${ }^{34}$.

Os exercícios diafragmáticos foram realizados em três séries de dez repetições, com as mãos das idosas posicionadas no abdômen, abaixo de suas últimas costelas, realizando uma inspiração profunda, direcionando o fluxo de ar para a região basal dos pulmões, onde o diafragma foi estimulado a vencer a pressão contrária realizada pelas mãos ${ }^{31}$.

$\mathrm{Na}$ inspiração fracionada em tempos, com expiração freno-labial, foi solicitado às idosas que realizassem três inspirações, em pequenas pausas, até que o pulmão ficasse completamente cheio, mantendo-se assim por dois segundos e, de forma lenta, com os lábios franzidos, expulsassem todo o ar inalado, sendo realizadas três séries de dez repetições ${ }^{32}$.

Os exercícios de ventilação padrão, com expiração curta, foram realizados pelas idosas em ciclos de inspiração, intercalados com pequenas expirações. As idosas realizaram uma inspiração profunda e expiraram uma pequena quantidade de ar (fase 1), novamente, outra pequena quantidade de ar (fase 2) e, por último, o esvaziamento residual. Foram realizadas duas séries de cinco repetições ${ }^{31}$.

Os exercícios respiratórios lúdicos foram realizados pelas idosas durante 10 minutos, na posição ortostática, utilizando bexigas da marca São Roque ${ }^{\circledR}$, modelo lisa, cor vermelha, número 7 , e línguas de sogra da marca Della-Cruz ${ }^{\circledR}$, cores sortidas. Foi observada a maneira que elas realizavam o sopro, pois não podiam distender as bochechas de ar durante o exercício ${ }^{33}$. Após os exercícios, realizaram novamente a espirometria e foram coletados os sinais vitais que, após serem verificados, 
não apresentando alterações hemodinâmicas, as participantes eram liberadas.

$\mathrm{Na}$ oitava visita, com duração de 60 minutos, as participantes realizaram a última coleta de sinais vitais, medidas da espirometria e cirtometria, para análise e comparação dos dados entre as visitas de número 01 a 08 e receberam informações quanto ao retorno dos pesquisadores às instituições, para apresentarem os resultados da pesquisa.

Os exercícios foram em séries, não cronometrados, ministrados por um único pesquisador. Por isso, as idosas puderam realizá-los com calma, evitando a fadiga muscular, realizando a ingestão de líquidos 2 horas antes do início do protocolo de exercícios e durante a execução do mesmo, evitando a desidratação e o ressecamento das mucosas das vias aéreas superiores ${ }^{35}$. Todas as atividades do estudo foram supervisionadas pelos pesquisadores, para que não ocoresse aspiração do bucal do espirômetro, da língua de sogra e do pó das bexigas. As bexigas foram lavadas em água corrente antes da realização dos exercícios, para retirada do pó.

Os dados referentes ao nome, à idade e ao estado de saúde das participantes foram registrados no QERC, sendo utilizado o Questionário de triagem pré-participação de saúde/ aptidão física relacionada à saúde, modificado do American College of Sports Medicine e American Heart Association ${ }^{36}$, que identificou 16 indivíduos em risco cardiovascular elevado e 5 em risco médio. Durante a execução dos exercícios, foram verificados os sinais vitais e observado a expressão facial das participantes, minimizando assim, os riscos de intercorrências hemodinâmicas.

As informações referentes aos sinais vitais ( $\mathrm{FC}, \mathrm{SaO}_{2}, \mathrm{FR}$ e PA), antes e após a realização do protocolo de treinamento com os exercícios respiratórios, foram anotadas em uma planilha, para registro e controle. Foi utilizado o oxímetro de pulso de dedo da marca Sportguard ${ }^{\circledR}$, esfigmomanômetro da marca G-TECH ${ }^{\circledR}$ e estetoscópio da marca G-TECH ${ }^{\circledR}$. A frequência respiratória foi coletada por meio da observação e da ausculta pulmonar, no tempo de 1 minuto, utilizando o estetoscópio da marca G-TECH ${ }^{\circledR}$ e o cronômetro da marca Taksun $^{\circledR}$. Durante os exercícios, as idosas não apresentaram desconforto e alterações hemodinâmicas fora dos padrões de normalidade ${ }^{34}$.

\section{Avaliação da Cirtometria}

A avaliação da mobilidade tóracoabdominal foi executada após consentimento verificado no TCLE, por meio da cirtometria ${ }^{12,13}$, com uma fita escalonada em centímetros da marca Worker ${ }^{\circledR}$, modelo fibra de vidro (95\% poliéster e $5 \%$ fibra de vidro), comprimento de $150 \mathrm{~cm}$, e largura de $16,0 \mathrm{~mm}$ e uma caneta dermatográfica marca Aspen ${ }^{\circledR}$, sendo realizada com base na descrição de Pedrini et al. ${ }^{37}$, de forma individual, onde a idosa permaneceu sem blusa e roupa íntima (soutien), sendo solicitado que ficasse em pé, com os membros superiores ao longo do corpo. Foram medidas as circunferências de três pontos anatômicos na seguinte ordem: prega axilar, processo xifóide e altura média entre o processo xifóide e cicatriz umbilical (abdominal), nos momentos de inspiração máxima e expiração máxima.

A diferença entre as medidas obtidas na inspiração e na expiração máximas em cada nível anatômico foi considerada como a mobilidade tóracoabdominal de cada região aferida. Todas as medidas foram realizadas 
pelo mesmo avaliador, e cada medida foi repetida três vezes em todos os níveis anatômicos, considerando a média ${ }^{37}$ entre os valores obtidos nas visitas de número 01 (antes do protocolo de exercícios) e 08 (após o protocolo de exercício), sendo anotados os valores em planilhas para controle, análise e comparação.

\section{Avaliação do PFE}

A coleta das medidas do PFE foi obtida por meio do espirômetro Peak Flow meter, da marca Medicate ${ }^{\circledR}$, com intervalo de medida entre zero a $900 \mathrm{~L} / \mathrm{min}$, em um ambiente arejado e mensurada por apenas um pesquisador em todas as visitas, antes e após os exercícios respiratórios, onde a idosa foi orientada a permanecer na posição sentada em $90^{\circ}$, numa cadeira, com os pés encostados, apoiados ao chão e a cabeça voltada para frente, não sendo permitido flexionar o pescoço e fazendo uso de um clip nasal, para evitar o escape de ar pelas narinas ${ }^{14,15}$. Foi solicitada uma inspiração profunda e, em seguida, uma expiração total, de forma rápida, no bucal do aparelho que foi posicionado entre os lábios da idosa. O procedimento foi realizado três vezes e foi obtida a média desses valores ${ }^{15}$.

Os valores das três medidas e a média do PFE, coletados nas visitas de número 01 (antes do protocolo de exercícios), 02 a 07 (durante o protocolo) e 08 (após o protocolo dos exercícios) foram anotados em planilhas para controle, análise e comparação.

\section{RESULTADOS}

O estudo foi composto por uma amostra de 21 mulheres $(74,71 \pm 6,47$ anos), que foram divididas em dois grupos, sendo estes, um grupo composto por idosas não institucionalizadas $(n=14)$ e outro composto por idosas institucionalizadas $(n=7)$.

As variáveis entre os grupos foram comparadas por meio das médias e dos desvios-padrões para a idade e o IMC no grupo de idosas não institucionalizadas $(71,67 \pm 4,97$ anos; $31,18 \pm 4,91 \mathrm{Kg} / \mathrm{m} 2$ ) e o grupo de idosas institucionalizadas $(80,86 \pm 1,69$ anos; $27,25 \pm 9,92 \mathrm{Kg} / \mathrm{m} 2$ ). Os PFE entre os grupos foram comparados por meio das médias e dos desvios-padrões, antes e após os exercícios, no grupo de idosas não institucionalizadas $(189,97 \pm 65,61 \mathrm{l} / \mathrm{min}$ vs $320,44 \pm 63,76 \mathrm{l} / \mathrm{min})$ e no grupo de idosas institucionalizadas $(172,34 \pm 79,64 \mathrm{l} / \mathrm{min}$ vs $249,95 \pm 55,38 \mathrm{l} / \mathrm{min})$.

Para a comparação das médias da idade e do IMC entre os dois grupos, foi aplicado o teste $t$ de Student, que não identificou diferença significativa. Os dados estão descritos a seguir, na Tabela 01.

Com o objetivo de avaliar as diferenças entre as medidas obtidas dos PFE, pré vs pós a realização dos exercícios respiratórios, as médias e os desvios-padrões foram comparados no grupo de idosas não institucionalizadas $(189,97 \pm 65,61 \mathrm{l} / \mathrm{min}$ vs $320,44 \pm 63,76 \mathrm{l} /$ min) e no grupo de idosas institucionalizadas $(172,34 \pm 79,64 \mathrm{l} / \mathrm{min}$ vs $249,95 \pm 55,38 \mathrm{I} / \mathrm{min})$, por meio do ANOVA de duas entradas, com medidas repetidas no fator tempo. O teste de post hoc de Bonferroni apresentou diferenças significativas de PFE $(p<0,05)$. Os dados estão abaixo, no Gráfico 01.

Os dados para a cirtometria torácica foram comparados por das médias e dos desvios-padrões entre a primeira e a oitava visitas respectivamente, antes e após a intervenção, sendo realizadas as circunferências torácicas 
Tabela 01. Descritiva das variáveis e do Pico de Fluxo Expiratório/PFE

\begin{tabular}{lccccccc}
\hline & \multicolumn{3}{c}{$\begin{array}{c}\text { Não Institucionalizadas } \\
(\mathrm{n}=14)\end{array}$} & \multicolumn{4}{c}{ Institucionalizadas } \\
$(\mathrm{n}=7)$
\end{tabular}

Fonte: Dados da pesquisa. Legenda: PFE (Pico de Fluxo Expiratório); DP (Desvio padrão); IMC (Índice de Massa Corpórea).

Gráfico 01. Comportamento do PFE antes e após os exercícios respiratórios das idosas Não Institucionalizadas e Institucionalizadas

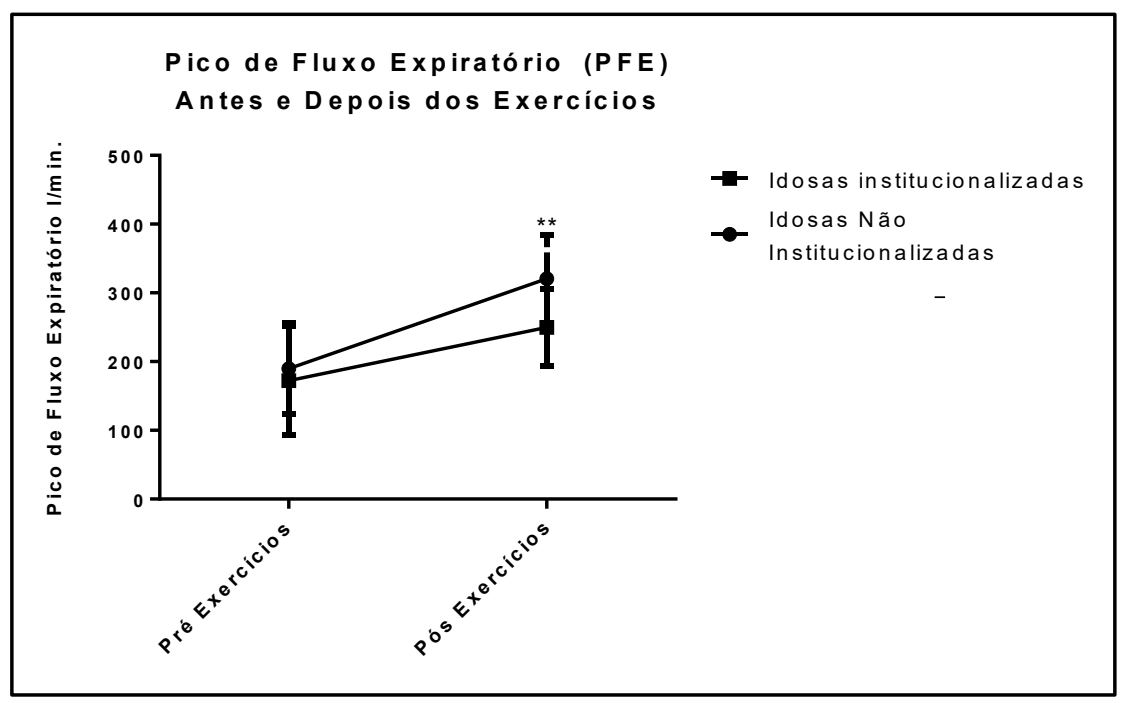

Fonte: Dados da pesquisa | * $(p<0,05)$

nos pontos anatômicos: axilar, xifoideano e abdominal, nas fases inspiratórias e expiratórias.

As diferenças das médias das medidas obtidas da cirtometria torácica pré vs pós a realização dos exercícios respiratórios do dia 01 ao dia 08 , foram comparadas entre os grupos por meio de uma ANOVA de duas entradas, com medidas repetidas no fator tempo, que não identificou diferenças significativas $(p>0,05)$.

$\mathrm{Na}$ análise intragrupos, as medidas das fases inspiratórias e expiratórias, antes e 
depois, foram analisadas pelo teste $T$ independente que apontou diferenças significativas da cirtometria torácica $(p<0,05)$, com as menores médias para o Grupo das Idosas Institucionalizadas (GII) e maiores para as Idosas não Institucionalizadas (GINI).

Para as medidas axilares inspiratórias, os valores antes e depois do GINI $(97,86 \pm 6,19$ vs $97,55 \pm 8,03 \mathrm{~cm})$ e do Gll $(94,67 \pm 15,8$ vs $95,05 \pm 16,42 \mathrm{~cm})$. Nas fases expiratórias do GINI $(96,26 \pm 6,39$ vs $95,54 \pm 7,69 \mathrm{~cm})$ e GII $(93,29 \pm 11,19$ vs $93,3 \pm 16,9 \mathrm{~cm})$. Para o nível xifoideano da cirtometria foi realizada a média nas fases inspiratórias do GINI $(90,72 \pm 7,2$ vs $91,47 \pm 7,33 \mathrm{~cm})$ e Gll $(92,15 \pm 18,19$ vs $89,32 \pm 18,72 \mathrm{~cm})$ e nas fases expiratórias do GINI $(90,4 \pm 7,44$ vs $90,41 \pm 7,5 \mathrm{~cm})$ e GII $(88,61 \pm 18,44$ vs $88,17 \pm 19,28 \mathrm{~cm})$.

E por fim, o nível abdominal nas fases inspiratórias do GINI $(98,46 \pm 12,61$ vs $96,3 \pm 10,58 \mathrm{~cm})$ e GII $(96,48 \pm 22,45$ vs $96,7 \pm 22,03 \mathrm{~cm}$ ). Nas fases expiratórias do GINI $(100,1 \pm 12,4$ vs $98,73 \pm 14,91 \mathrm{~cm})$ e GII $(96,3 \pm 22,57$ vs $96,37 \pm 22,17 \mathrm{~cm})$. As diferenças foram significativas na fase inspiratória entre os grupos. Entretanto, para a fase expiratória, a circunferência abdominal não apresentou diferenças intragrupos e nenhuma das duas fases apresentou diferença no fator tempo.

\section{DISCUSSÃO}

Os resultados do atual estudo indicaram que o programa de exercícios respiratórios, durante quatro semanas, promoveu melhora do PFE em idosas. Estes achados foram encontrados nos estudos de Barros et al. ${ }^{38}$, que realizaram treinamento muscular inspiratório durante internação de pacientes submetidos à cirurgia de revascularização do miocárdio e, também, de Durante et al. ${ }^{39}$, que avaliaram os efeitos de um programa de treinamento muscular inspiratório domiciliar (TMID) sobre a função pulmonar em seis semanas.

Ladosky, Botelho e Júnior ${ }^{40}$ constataram que o PFE é menor em indivíduos obesos. Essa associação entre PFE vs IMC também foi observada nos estudos de Lisboa et al. ${ }^{20}$, onde verificaram redução dessas variáveis no grupo de idosas institucionalizadas. Porém, Matteoni et al. ${ }^{41}$, ao avaliarem mulheres obesas, notaram que o programa de treinamento físico causou aumento significativo no PFE e Freitas et al. ${ }^{8}$ concluíram que o PFE foi mais elevado nos idosos ativos em relação aos moderadamente ativos e, no presente estudo, o grupo de idosas não institucionalizadas mostrou o IMC e o PFE maiores, em relação ao grupo de idosas institucionalizadas.

Paes et al. ${ }^{42}$ compararam os valores de PFE, de uma amostra da Cidade de São Carlos (SP), com valores de amostras internacionais, que resultou na diferença significativa de sua amostra, na faixa etária de 61 a 70 anos. Embora, a idade avançada seja uma variável associada a baixos valores de PFE e a cognição, para Almeida et al. ${ }^{43}$ e Yassuda et al. ${ }^{44}$ é possível que as condições de saúde física influenciem mais nesses valores do que a idade propriamente dita. $E$ isso foi demonstrado contrariamente no presente estudo, pois o grupo de idosas institucionalizadas apresentou idades mais elevadas e menores pontuações no QERC, comparado ao grupo de idosas não institucionalizadas.

Por outro lado, na avaliação da mobi- 
lidade tóracoabdominal, Jesus et al ${ }^{45}$ encontraram resultados positivos na cirtometria de mulheres adultas (50-55 anos) que praticaram o método Pilates, resultando no aumento da complacência torácica, da força muscular e da flexibilidade. Em contrapartida, estudos ${ }^{6,30,46}$ salientam que a redução da complacência torácica pode estar presente aos 50 anos, porém, mais evidente aos 80 anos. Apesar do presente estudo não ter apresentado dados significativos para a expansão ou retração torácica nos pontos axilar, xifoideano e abdominal da cirtometria, Ruivo et al. ${ }^{6}$ explicaram que a diminuição da expansibilidade torácica decorre da redução da distensão do sistema toracopulmonar, que está diretamente ligada à idade cronológica.

Mas, Colman e Beraldo ${ }^{47}$, ao estudarem pacientes lesados medulares com disfunções pulmonares, observaram que o treinamento com incentivadores respiratórios, após 30 sessões, é capaz de gerar aumento significativo da força muscular dos intercostais e do diafragma. Sob outra perspectiva, França et al. ${ }^{48}$, em seu estudo, identificaram aumento significativo da força muscular em 12 sessões e Rodrigues et al. ${ }^{49}$ ao avaliarem os efeitos de um programa de exercícios, para readequação do complexo toracopulmonar e qualidade de vida em pacientes com DPOC, verificaram aumento significativo da expansibilidade torácica na região xifoideana e abdominal, após 12 semanas de tratamento.

Talvez por isso, não foram encontradas, neste estudo, alterações da cirtometria torácica com os exercícios, devido ao número reduzido de intervenções.

As participantes deste estudo mos- traram-se incomodadas com a realização do método da cirtometria, sem vestimentas, na região tóracoabdominal, o que é apresentado e justificado no estudo de Fin et al. ${ }^{50}$, como processo de julgamento corporal, em que a idosa julga o seu corpo, com base nos padrões ditados pela sociedade, como sinônimo de beleza. Este procedimento foi motivo de desistência de uma das voluntárias deste estudo.

Não foram encontrados estudos na literatura, relacionando aumento do PFE com cirtometria torácica, proveniente de exercícios respiratórios. Acredita-se que o aumento do PFE, em ambos os grupos, tenha sido resultante do protocolo de exercícios, enquanto, a cirtometria não apresentou diferença significativa, devido à diminuição da complacência torácica, que é resultante das alterações anatômicas e fisiológicas que acontecem no envelhecimento $0^{6,9,50,51}$, além do pequeno período de treinamento muscular, visto que não foram encontrados na literatura, estudos que abordassem o treinamento muscular respiratório, com tempo inferior a 12 sessões.

\section{CONCLUSÃO}

O PFE dos grupos de idosas institucionalizadas e não institucionalizadas aumentou durante o programa de exercícios respiratórios em quatro semanas. Entretanto, para os valores da cirtometria torácica não ocorreram alterações e as idosas institucionalizadas apresentaram médias de expansão e de retração torácica menores do que as não institucionalizadas. 


\section{REFERÊNCIAS}

1. Lustosa LP, Oliveira LA, Santos LS, Guedes RS, Parentoni AN, Pereira LSM. Efeito de um programa de treinamento funcional no equilíbrio postural de idosas da comunidade. Fisioter Pesq. 2010;17(2):153-156.

2. Fernandes AMBL, Ferreira JJA, Stolt LROG, Brito GEG, Clementino ACCR, Sousa NM. Efeitos da prática de exercício físico sobre o desempenho da marcha e da mobilidade funcional em idosos. Fisioter Mov. 2012; 25(4):821-830.

3. Souza CC, Valmorbida LA, Oliveira JP, Borsatto AC, Lorenzini M, Knorst MR, Melo D, Creutzberg M, Resende TL. Mobilidade funcional em idosos institucionalizados e não institucionalizados. RBGG. 2013;16(2):285-293.

4. Mynarsk J, Santos L, Verffel A, Mello D, Berticell MW, Olkoski MM. Efeitos de diferentes programas de exercícios físicos sobre a composição corporal e a autonomia funcional de idosas com risco de fratura. Rev Educ Fís UEM. 2014; 25(4):609-618.

5. Lopes EDS, Ruas G, Patrizzi LJ. Efeitos de exercícios do método Pilates na força muscular respiratória de idosas: um ensaio clínico. RBGG. 2014; 17(3):517-523.

6. Ruivo S, Viana P, Martins C, Baeta C. Efeito do envelhecimento cronológico na função pulmonar: Comparação da função respiratória entre adultos e idosos saudáveis. Rev Port Pneumol. 2009; 15(4):629-653.

7. Santos TC, Travensolo CF. Comparação da força muscular respiratória entre idosos sedentários e ativos: estudo transversal. Kairós. 2011; 14(4):107-121.

8. Freitas FS, Ibiapina CC, Alvim CG, Britto RR, Parreira VF. Relação entre força de tosse e nível funcional em um grupo de idosos. RBF. 2010;14(6):470-476.

9. Oliveira M, Santos CLS, Oliveira CF, Ribas DIR. Efeitos da técnica expansiva e incentivador respiratório na força da musculatura respiratória em idosos institucionalizados. Fisioter Mov. 2013; 26(1):133-140.

10. Andrade FSSD, Junior JAF, Simões MS, Menezes PDG, Sousa FJD, Teixeira RC, et al. Análise comparativa de pico de fluxo expiratório entre idosos sedentários e praticantes de atividade física. GGAGING. 2014; 8(4):211-215.
11. Buchman BAS, Boyle PA, Wilson RS, Liping Gu, Bienias $\mathrm{JL}$, Bennett DA. Pulmonary Function, Muscle Strength and Mortality in Old Age. Mech Ageing Dev. 2008;129(11):625631.

12. Baltieri L, Santos LA, Furlan GN, Moreno MA. Respiratory muscle strength and thoracoabdominal mobility in sedentary elderly, adults and players of adapted volleyball: a pilot study. Fisioter Pesq. 2014; 21(4):314-319.

13. Bezerra RO, Júnior FFUS, Campos NG. Análise da mobilidade da caixa torácica e da força muscular respiratória em adultos jovens. Revista Digital. 2012;17(175):1-9.

14. Costa D, Jamami M. Bases Fundamentais da Espirometria RBF. 2001; 5(2):95-102.

15. Miller MR, Hankinson J, Brusasco V, Burgos F, Casaburi R, Coates A, et al. Standardisation of spirometry. Eur Respir J. 2005; 26(2):319-338.

16. Pettenon R, Milano D, Bittencourt DC, Schneider RH Adaptação funcional do aparelho respiratório e da postura no idoso. RBCEH. 2008; 5(2):64-77.

17. Machado FA, Kravchychyn ACP, Peserico CS, Silva DF, Mezzaroba PV. Reprodutibilidade do desempenho em provas de corrida de 5 e $10 \mathrm{~km}$ em pista de atletismo. RBCE. 2015;37(3):207-213.

18. Instituto Brasileiro de Geografia e Estatística-IBGE. Informações Estatísticas. 2017.

19. Fonseca $\mathrm{NT}$, Contato $\mathrm{C}$. Análise da mecânica respiratória antes e após o uso do threshold em indivíduos idosos. Saúde UNIPAM. 2010; 1(2):101-108.

20. Lisboa APAZ, Meereis ECW, Gonçalves MP, Silva AMV. Análise comparativa entre idosos ex-tabagistas institucionalizados e não institucionalizados quanto à função respiratória, níveis de ansiedade, de depressão e de qualidade de vida. Kairós. 2013; 16(4):65-77.

21. Caldeira JB, Junior NAL, Sancho AG, Rosa JLS, Faria D, Balthazar MC. Avaliação do pico de fluxo expiratório em idosos autônomos institucionalizados e não institucionalizados. Fisioter Bras. 2012; 13(4):272-276. 
22. Junior LAF, Rubleski A, Garcia D, Tieppo J, Vercelino R, Dal Bosco A, Monteiro MB, Dias AS. Avaliação da Força Muscular Respiratória e da Função Pulmonar em Pacientes com Insuficiência Cardíaca. Arq Bras Cardiol. 2007; 89(1):36-41.

23. Freitas ERFS, Araujo ECLS, Alves KS. Influência do tabagismo na força muscular respiratória em idosos. Fisioter Pesq. 2012;19(4):326-331.

24. Thomé JS, Olmedo L, Santos FM, Magnani KL, Müller PT, Christofoletti G. Pacientes com doença de Parkinson sob assistência fisioterapêutica apresentam parâmetros pulmonares melhores do que controles sedentários. Fisioter Pesq. 2016; 23(1):30-37.

25. Fonseca MA, Cader SA, Dantas EHM, Bacelar SC, Silva EB, Leal SMO. Programas de treinamento muscular respiratório: impacto na autonomia funcional de idosos. RAMB. 2010; 56(6):642-648.

26. Pascotini FS, Ramos MC, Silva AMV, Trevisan ME. Espirometria de incentivo à volume versus a fluxo sobre parâmetros respiratórios em idosos. Fisioter Pesq. 2013; 20(4):355-360

27. Yamaguti WPS, Sakamoto ET, Panazzolo D, Peixoto CC, Cerri GG, Albuquerque ALP. Mobilidade diafragmática durante espirometria de incentivo orientada a fluxo e a volume em indivíduos sadios. J Bras Pneumol. 2010; 36(6):738-745

28. Costa D, Gonçalves HA, Lima LP, Ike D, Cancelliero KM, Montebelo MIL. Novos valores de referência para pressões respiratórias máximas na população brasileira. J Bras Pneumol. 2010; 36(3):306-312.

29. Etemadinezhad S, Alizadeh A. Valores de referência para espirometria em adultos saudáveis na província de Mazandaran, Irã. J Bras Pneumol. 2011; 37(5):615-620.

30. Slongo M, Morsch ALBC. Comportamento das pressões respiratórias máximas após um programa de fisioterapia respiratória em idosos institucionalizados. FisiSenectus. 2015; 3(1):39-49.

31. Bom EA, Souza CV, Thiesen RAS, Miranda ECM, Souza CA. Avaliação das condições respiratórias na fase inicial do transplante de células-tronco hematopoiéticas. RBHH. 2012; 34(3):188-192.
32. Lima EVNCL, Lima WL, Nobre A, Santos AM, Brito LMO, Costa MRSR. Treinamento muscular inspiratório e exercícios respiratórios em crianças asmáticas. J Bras Pneumol. 2008; 34(8):552-558

33. Reis JRG, Nascimento LCG, Ramos JM, Quemelo PRV, Filho DMP, Neiva CM et al. Programa de exercícios respiratórios com sessões de curta duração e os efeitos na mecânica ventilatória de crianças com asma. Revista Digital. 2015;20(211):1-9.

34. Simão AF, Précoma DB, Andrade JP, Correa Filho $\mathrm{H}$, Saraiva JFK, Oliveira GMM et al. Sociedade Brasileira de Cardiologia. I Diretriz Brasileira de Prevenção Cardiovascular. Arq Bras Cardiol. 2013; 101(Supl.2):1-63.

35. Hernandez AJ, Nahas RM, Rodrigues T, Meyer F, Zogaib $\mathrm{P}$, Lazzoli JK, et al. Modificações dietéticas, reposição hídrica, suplementos alimentares e drogas: comprovação de ação ergogênica e potenciais riscos para a saúde. RBME. 2009;15(3):03-12.

36. Kaminsky LA. ACSM's Health-Related Physical Fitness Assessment Manual. 3. ed. Philadelphia: Lippincott Williams \& Wilkins. 2010.

37. Pedrini A, Gonçalves MA, Leal BE, Yamaguti WPS, Paulin E. Comparação entre as medidas de cirtometria tóraco-abdominal realizadas em decúbito dorsal e em ortostatismo. Fisioter Pesq. 2013; 20(4):373-378.

38. Barros GF, Santos CS, Granado FB, Costa PT, Límaco RP, Gardenghi G. Treinamento muscular respiratório na revascularização do miocárdio. BJCVS. 2010; 25(4):483-490.

39. Durante A, Rodigheri A, Rockenbach CWF, Pimentel GL, Leguisamo CP, Calegari L. Treinamento muscular inspiratório melhora a força muscular respiratória e o pico de fluxo expiratório em idosas hipertensas. ConScientiae Saúde. 2014;13(3):364-371.

40. Ladosky W, Botelho MAM, Júnior JPA. Chest mechanics in morbidly obese non-hypoventilated patients. Respir Med. 2011; 95(4):281-286.

41. Matteoni SPC, Júnior CRB, Teixeira LR. Efeito de um programa de condicionamento físico no broncoespasmo induzido pelo exercício em mulheres obesas. RBME. 2009;15(3):190-194. 
42. Paes CD, Pessoa BV, Jamami M, Lorenzo VAPD, Marrara KT. Comparação de valores de PFE em uma amostra da população da cidade de São Carlos, São Paulo, com valores de referência. J Bras Pneumol. 2009; 35(2):151-156.

43. Almeida MHM, Beger MLM, Watanabe HAW. Oficina de memória para idosos: estratégia para promoção da saúde. Interface. 2007;11(22):271-280.

44. Yassuda MS, Batistoni SST, Fortes AG, Neri AL. Treino de memória no idoso saudável: Benefícios e Mecanismos. PRC. 2006;19(3):470-481.

45. Jesus LT, Baltieri L, Oliveira LG, Angeli LR, Antonio SP, Forti EMP. Efeitos do método Pilates sobre a função pulmonar, a mobilidade Tóracoabdominal e a força muscular respiratória: ensaio clínico não randomizado, placebo-controlado. Fisioter Pesq. 2015 ;22(3):213-222.

46. Pascotini FS, Fedosse E, Ramos MC, Ribeiro VV, Trevisan ME. Força muscular respiratória, função pulmonar e expansibilidade Tóracoabdominal em idosos e sua relação com o estado nutricional. Fisioter Pesq. 2016; 23(4):416-422.

47. Colman ML, Beraldo PC. Estudo das variações de pressão inspiratória máxima em tetraplégicos, tratados por meio de incentivador respiratório, em regime ambulatorial. Fisioter Mov. 2010; 23(3):439-49.
48. França NC, Filho ASB, Cunha EC, Silva CMS, Cordeiro ALL. Efeitos do Treinamento Muscular Inspiratório Associado ao Aeróbio na Capacidade Funcional em Pacientes com Insuficiência Cardíaca Crônica. RPF. 2015; 5(2):108-113.

49. Rodrigues CP, Alves LA, Matsuo T, Gonçalves CG, Hayashi D. Efeito de um programa de exercícios direcionados à mobilidade torácica na DPOC. Fisioter Mov. 2012; 25(2):343-349.

50. Fin TC, Portella MR, Scortegagna AS. Velhice e beleza corporal das idosas: conversa entre mulheres. RBGG. 2017; 20(1):77-87.

51. Hochhegger B, Meirelles GS, Irion K, Zanetti G, Garcia E, Moreira J, Marchiori E. O tórax e o envelhecimento: manifestações radiológicas. J Bras Pneumol. 2012; 38(5):656-665.

\section{CORRESPONDÊNCIA}

Wagner Elias de Melo Moreira

Rua Aracy Sampaio, $n^{\circ} 26$, Centro

Dores de Campos, Minas Gerais, Brasil.

36213-000

E-mail: wagner.fisioterapia@yahoo.com.br 